\title{
TESTING SEIBERG-WITTEN SOLUTION
}

HUTP-97/A102, ITEP-TH-74/97, hep-th/9801061

To appear in the Proceedings of Cargese conference, June 1997

\author{
A. LOSEV \\ Institute of Theoretical and Experimental Physics, \\ 117259, Moscow, Russia \\ N. NEKRASOV \\ Institute of Theoretical and Experimental Physics, \\ 117259, Moscow, Russia \\ Lyman Laboratory of Physics, Harvard University, \\ Cambridge MA 02138
}

AND

S. SHATASHVILI

Department of Physics, Yale University, New Haven CT 06520, Box 208120

on leave of absence from

St. Petersburg Steklov Mathematical Institute

\begin{abstract}
We propose a few tests of Seiberg-Witten solutions of $\mathcal{N}=2$ supersymmetric gauge theories by the instanton calculus in twisted gauge theories. We re-examine the low-energy effective abelian theory in the presence of sources and present the formalism which makes duality transformations transparent and easily fixes all the contact terms in a broad class of theories. We also discuss ADHM integration and its relevance to the stated problems.
\end{abstract}

\section{Introduction and summary}

One of the sources of the recent duality revolution in string theory is the solution of N. Seiberg and E. Witten [1] of $\mathcal{N}=2$ supersymmetric Yang-Mills theory which has been tested in many indirect ways but never directly. The solution, among other things, predicts the formula for the effective coupling constant of the low-energy theory as a function of the order parameter $u$ 
(in $S U(2)$ theory):

$$
\tau(u)=\frac{2 i}{\pi} \log \left(\frac{u}{\Lambda^{2}}\right)+\sum_{n=1}^{\infty} \tau_{n}\left(\frac{\Lambda^{2}}{u}\right)^{2 n}
$$

The coefficients $\tau_{n}$ are claimed to be instanton corrections. The direct test of (1) would involve integration of a certain form over the moduli space of instantons on $\mathbb{R}^{4}$. This integration can be shown to localize onto the space of point-like instantons and has a potential divergence in it related to the fact that the space of point-like instantons is non-compact (it is a resolution of singularities of $S^{k} \mathbb{R}^{4}$ ). Although this difficulty is avoidable by appropriate regularization so far no substantial success has been achieved on this route (nevertheless, see the related discussion in [5]). The infrared regularization of the instanton integrals turns out to be tricky problem. At first sight it is possible to simply put the periodic boundary conditions and study the theory on a four-torus. Unfortunately the puriest test of (1) would involve the computation of the correlation functions of observables which preserve some supersymmetry which are called topological correlation functions. TCF's are almost insensitive to the geometry of the moduli space of vacua of the gauge theory in the case, where the four-manifold $X$ has $b_{2}^{+}>1$. To make the TCF probe the geometry of the moduli space of vacua one must find such a manifold $X$ which allows for the instanton calculus to be perfomed and has $b_{2}^{+}=1$. The latter constraint will be explained below. Examples of such manifold $X$ are provided by ruled surfaces, $X=S^{2} \times S^{2}$ among them . The manifold $X$ does not have any covariantly constant spinors and the original supersymmetric theory has no conserved charges. One may study a twisted theory, introduced by E. Witten in [13]. The latter has more freedom in the choice of action then the original physical theory. In particular, one may take the limit of zero coupling in the non-abelian theory which reduces the computations of TFC to the counting intersection numbers of homology classes of compactified moduli space of instantons ion $X$. Such a counting problem is equivalent to the way S. Donaldson formulated his invariants in the language of problems of "generic position" (for the gauge groups $S U(2)$ and $S O(3)$ ). The insight coming from the equivalence of Donaldson theory and Witten's twisted version of $\mathcal{N}=2$ super-Yang-Mills theory is that the same problem may be addressed in the infrared limit. It seems that the well-defined ultraviolet problem in the field-theoretic formulation involves the definition of the compactified moduli space of distinct points on the space-time manifold $X$. The infrared theory contains the information about this compactification in the contact terms between the observables . In the context of gauge theory these contact terms can be studied using severe constraints of modular invariance and ghost number anomalies . 


\section{The micro/macroscopic theories}

The $\mathcal{N}=2$ super-Yang-Mills theory with the gauge group $G$ has as the basic field the vector multipler of four dimensional $\mathcal{N}=2$ supersymmetry, which involves a gauge field $A^{a}$, a complex scalar $\phi^{a}$ and a pair of Weyl fermions $\psi_{\alpha}^{a}, \lambda^{\dot{\alpha}, a}$. All fields are in the adjoint representation of the gauge group. For the sake of convenience we study the twisted version of the theory where fermions have different Lorentz spins. One should understand once and for all that as long as "physical" questions are asked about the theory formulated on $\mathbb{R}^{4}$ (euclidean signature) there is no way to distinguish the two models. The distinction appears when the curved gravitational background appears. The twisted theory always has at least one conserved supercharge $Q$. The square of $Q$ is an infinitesimal gauge transformation with parameter $\phi$. The operator $Q$ acts on the Hilbert space of the theory. Its square vanishes on the physical Hilbert space formed by the gauge invariant states. The Hilbert space $\mathcal{H}_{S}$ is obtaned by quantizing the space of fields on a three-fold $S$. The Hamiltonian of the theory can be represented as a $Q$-commutator:

$$
H=\left\{Q, G_{r}\right\}
$$

where $G_{r}$ is a certain operator which is in fact a twisted version of one of the eight supercharges of the original $\mathcal{N}=2$ susy. There is an operator of ghost number $U$ which can be represented as the integral of the corresponding current $J: U=\int_{S} J$. The Hamiltonian has ghost charge 0 , the operator $Q$ has $U=1, G_{r}$ has $U=-1$, the fields have the following charges:

TABLE 1. Ghost charges, Lorentz spins, $Q$ action

\begin{tabular}{lllll}
\hline Field & $S U(2)_{L} \times S U(2)_{R}$ & $U$ charge & Statistics & $Q$ action \\
\hline$A$ & $\left(\frac{1}{2}, \frac{1}{2}\right)$ & 0 & $\mathrm{~B}$ & $\psi$ \\
$\psi$ & $\left(\frac{1}{2}, \frac{1}{2}\right)$ & 1 & $\mathrm{~F}$ & $D_{A} \phi$ \\
$\phi$ & $(0,0)$ & 2 & $\mathrm{~B}$ & 0 \\
$\chi$ & $(0,1)$ & -1 & $\mathrm{~F}$ & $H$ \\
$H$ & $(0,1)$ & 0 & $\mathrm{~B}$ & {$[\phi, \chi]$} \\
$\eta$ & $(0,0)$ & -1 & $\mathrm{~F}$ & {$[\phi, \bar{\phi}]$} \\
$\bar{\phi}$ & $(0,0)$ & -2 & $\mathrm{~B}$ & $\eta$ \\
\hline
\end{tabular}

Since on the gauge invariant states $Q^{2}=0$ one may study the cohomology space $H_{S}=\operatorname{Ker} Q / \operatorname{Im} Q$. The importance of this space is that it is preserved under the evolution and changes of the metric. Indeed, if $Q\left|\psi_{0}\right\rangle=0$ and we identify the states in the evolved space with the help of 
Hamiltonian $H$ then

$$
\left|\psi_{t}\right\rangle=e^{-t H}\left|\psi_{0}\right\rangle=\left|\psi_{0}\right\rangle+Q\left|\psi_{t}^{\prime}\right\rangle
$$

that is the cohomology class of a state does not change with time. Now, having got the space $H_{S}$ one may wonder how many states are there and what are the natural operators which act on $H_{S}$. To answer the second question we must figure out what are the operators $\mathcal{O}$ the theory which obey $\{Q, \mathcal{O}\}=0$. The Hamiltonian is one of them but it acts trivially on $H_{S}$. What are non-trivial operators? Clearly, these are the ones which cannot be represented as $\{Q, \operatorname{smth}\}$, that is we are interested in $Q$-cohomology in operators.

The twisted gauge theory has various non-local observables. They are labelled by the following data: a gauge invariant function $\mathcal{P}$ on the Lie algebra $\mathbf{g}$ and the number $p=0, \ldots, 4$. The $p$-observable $\mathcal{O}_{\mathcal{P}}^{(p)}$ is a $p$-form and is supposed to be integrated over a $p$-cycle in the manifold $X$. The easiest way to write all $p$-observables at once is to expand the function $\mathcal{P}(\phi+\psi+F)$ is the forms of varios degree. The observables $\mathcal{O}^{(p)}$ then automatically obey the descend equations

$$
d \mathcal{O}^{(p)}=\left\{Q, \mathcal{O}^{(p+1)}\right\}
$$

Of course, the representative of $Q$-cohomology is not unique. The one we get by expanding $\mathcal{P}(\phi+\psi+F)$ is called holomorphic. For some reasons one may prefer a harmonic representative, which is obtained from $\mathcal{P}(\phi)$ by acting with $G_{\mu}$ on it. Here $G_{\mu}$ are the twisted supercharges, which obey $\{Q, G\}=d$ (we saw one of them in (2)). The holomorphic observables can be written quite explicitly: $\mathcal{O}_{\mathcal{P}}^{(0)}=\mathcal{P}(\phi), \mathcal{O}_{\mathcal{P}}^{(1)}=\frac{\partial \mathcal{P}}{\partial \phi^{a}} \psi^{a}, \mathcal{O}_{\mathcal{P}}^{(2)}=\frac{\partial \mathcal{P}}{\partial \phi^{a}} F^{a}+$ $\frac{1}{2} \frac{\partial^{2} \mathcal{P}}{\partial \phi^{a} \partial \phi^{b}} \psi^{a} \psi^{b}, \mathcal{O}_{\mathcal{P}}^{(3)}=\frac{1}{2} \frac{\partial^{2} \mathcal{P}}{\partial \phi^{a} \partial \phi^{b}} \psi^{a} F^{b}+\frac{1}{6} \frac{\partial^{3} \mathcal{P}}{\partial \phi^{a} \partial \phi^{b} \partial \phi^{c}} \psi^{a} \psi^{b} \psi^{c}$. The top degree observable equals:

$\mathcal{O}_{\mathcal{P}}^{(4)}=\frac{1}{2} \frac{\partial^{2} \mathcal{P}}{\partial \phi^{a} \partial \phi^{b}} F^{a} F^{b}+\frac{1}{3 !} \frac{\partial^{3} \mathcal{P}}{\partial \phi^{a} \partial \phi^{b} \partial \phi^{c}} F^{a} \psi^{b} \psi^{c}+\frac{1}{4 !} \frac{\partial^{4} \mathcal{P}}{\partial \phi^{a} \partial \phi^{b} \partial \phi^{c} \partial \phi^{d}} \psi^{a} \psi^{b} \psi^{c} \psi^{d}$

It enters the Seiberg-Witten low-energy effective action, where all the fields are specialized to be abelian. In general, the whole action $S$ equals the sum of the 4-observable, constructed out of the prepotential $\mathcal{F}$ and the $Q$-exact term:

$$
S=\int_{X} \mathcal{O}_{\mathcal{F}}^{(4)}+\{Q, R\}
$$


We can view all observables $\mathcal{O}_{\mathcal{P}}^{(p)}$ for all $\mathcal{P}$ and $p$ as deformations of the theory by adding them to the action (6) as follows:

$$
S=\sum_{p=0}^{4} T^{p, \alpha} \int_{X}\left(\mathcal{O}_{\mathcal{F}_{p}}^{(p)}+\left\{Q, R^{(p)}\right\}\right) \wedge e_{\alpha}
$$

where $e_{\alpha}$ runs through a basis in the cohomology group $H^{*}(X ; \mathbb{R})$. In turn, one may expand $T^{p, \alpha} \mathcal{F}_{p}=\sum_{k} T^{k, \alpha} \mathcal{P}_{k}$ with $\mathcal{P}_{k}$ running over a space of invariant polynomials.

The infrared theory is also a twisted supersymmetric gauge theory with abelian gauge group. The observable $\mathcal{O}_{\mathcal{P}, \text { UV }}^{(p)}$ of the ultraviolet theory flows to the observable $\mathcal{O}_{\mathcal{P} \text {,IR }}^{(p)}$ of the infrared theory. Since the gauge group of the infrared theory is the maximal torus (extended by the Weyl group) of the ultraviolet theory there is one-to-one correspondence between the gauge invariant functions $\mathcal{P}_{\mathrm{UV}}$ and $\mathcal{P}_{\mathrm{IR}}$. Now suppose that two cycles $C^{p} \in H_{p}(X)$ and $C^{q} \in H_{q}(X)$ intersect: $C_{p} \cap C_{q}=C_{p+q-4} \in H_{p+q-4}(X)$. No matter how large the scale is to get at the intersection point(s) we must look at very short distances. The contribution of the short distance physics is the contact term:

$$
\int_{C^{p}} \mathcal{O}_{\mathcal{P}, \mathrm{UV}}^{(p)} \int_{C^{q}} \mathcal{O}_{\mathcal{Q}, \mathrm{UV}}^{(q)} \longrightarrow \int_{C^{p}} \mathcal{O}_{\mathcal{P}, \mathrm{IR}}^{(p)} \int_{C^{q}} \mathcal{O}_{\mathcal{Q}, \mathrm{IR}}^{(q)}+\int_{C^{p+q-4}} \mathcal{O}_{\mathcal{C}(\mathcal{P}, \mathcal{Q}), \mathrm{IR}}^{(p+q-4)}
$$

The main problem of the effective usage of low-energy theory is the determination of the contact terms $\mathcal{C}(\mathcal{P}, \mathcal{Q})$. The important constraint on the function $\mathcal{C}(\mathcal{P}, \mathcal{Q})$ is that it is independent of $p$ and $q$. This property was called in [17] the universality of the contact terms. It follows from the simple fact that the relative geometry of a pair of points on a four-fold $X$ is independent of the dimensions of the cycles the points are confined to. This universality property implies some non-trivial idenities between the modular functions which we describe in the next section. These identities seem to distinguish Seiberg-Witten-like families of abelian varieties and serve as a test of SW technology.

The next contact term is the triple term : suppose three cycles $C^{p_{1}}, C^{p_{2}}, C^{p_{3}}$ are given, then the analogue of (8) is:

$$
\begin{gathered}
\prod_{i=1}^{3} \int_{C^{p_{i}}} \mathcal{O}_{\mathcal{P}_{i}, \mathrm{UV}}^{\left(p_{i}\right)} \longrightarrow \prod_{i=1}^{3} \int_{C^{p_{i}}} \mathcal{O}_{\mathcal{P}_{i}, \mathrm{IR}}^{\left(p_{i}\right)}+ \\
\sum_{(i, j, k)=(1,2,3)} \int_{C^{p_{i}} \cap C^{p_{j}}} \mathcal{O}_{\mathcal{C}\left(\mathcal{P}_{i}, \mathcal{P}_{j}\right), \mathrm{IR}}^{\left(p_{i}+p_{j}-4\right)} \int_{C^{p_{k}}} \mathcal{O}_{\mathcal{P}_{k}, \mathrm{IR}}^{p_{k}} \\
\quad+\int_{C^{p_{1}} \cap C^{p_{2}} \cap C^{p_{3}}} \mathcal{O}_{\mathcal{C}\left(\mathcal{P}_{1}, \mathcal{P}_{2}, \mathcal{P}_{3}\right), \mathrm{IR}}^{p_{1}+p_{2}}
\end{gathered}
$$


If the four-observables are involved then we get an infinite sequence of contact functions $\mathcal{C}\left(\mathcal{P}_{1}, \ldots, \mathcal{P}_{k}\right)$ which are nicely organized in the generating function $\mathcal{F}$ :

$$
\mathcal{F}(T)=\mathcal{F}_{0}+\sum_{k=1}^{\infty} T^{1} \ldots T^{k} \mathcal{C}\left(\mathcal{P}_{1}, \ldots, \mathcal{P}_{k}\right)
$$

where $T^{k}$ 's are the deformation parameters, which wil be referred to as times. The first order term in the function $\mathcal{F}$ is $T^{k} \mathcal{C}\left(\mathcal{P}_{k}\right)$. The "one-contact function" $\mathcal{C}\left(\mathcal{P}_{k}\right)$ is the function $\mathcal{P}_{k}$ itself.

The claim is finally:

The TCF (= partition function) in the theory with the action (7) is equal to that in the low-energy abelian theory with the action

$$
S=\int_{X} \mathcal{F}\left(a+\psi+F, T^{k, \alpha} e_{\alpha}\right)
$$

In [17] it is shown that the function $\mathcal{F}(T)$ has a very simple geometrical meaning. It describes the deformations of $\Gamma$-invaraint Lagrangian submanifolds in a complex vector symplectic space $\mathbb{C}^{2 r}$, where $\Gamma$ is a certain discrete subgroup of linear symplectic group.

Similarly to the studies of two dimensional type B topological sigma models a lot of information can be rather easily obtained by working with holomorphic representatives. This is the point of view taken in this lecture. The reader interested in harmonic theory should consult [16], [17], [28].

\section{Coulomb branch measure}

The general strategy in using the statement (11) is to compute the integral over the zero modes of the fields entering abelian multiplets. The final integral is the one over the scalar field $a$ and it involves various modular forms integrated over the modular domain. Without going into tremendous complications of the actual comutations in any realistic setting one may say a lot of non-trivial things about the measure itself. To compare the last sentence with the development of perturbative string theory one may say that the measure of stringy loop computations has been stduied extensively although the actual integral is known only in a limited number of instances.

First of all we must explain what distinguishes the manifolds with $b_{2}^{+}=$ 1. This issue is not possible to see working with holomorphic fields only. It turns out that the harmonic representative of the four-observable contains the only one term $\left(\int_{X} \eta \chi \wedge F\right)$ which couples to a zero mode of $\eta$ and survives in the limit of very large $X$. Thus one needs presicely one zero mode of the field $\chi$ otherwise the effective measure vanishes as an inverse power of the size of manifold $X$. 
It turns out that the TCF's are not exactly topological invariants. The reason is that sometimes the enumerative problem of Donaldson's is not well-behaved under the variations of parameters, such as the metric. This occurs precisely when $b_{2}^{+} \leq 1$ due to the abelian instantons ${ }^{1}$. The jumps of the correlations functions/Donaldson invariants for $b_{2}^{+}=1$ are under control [22], [23],[24],[16].

Below we describe a few techinques of computing the pair contact terms in various theories with simply-laced gauge groups ${ }^{2}$. We use the low-energy effective theory, whose action on $\mathbb{R}^{4}$ has been computed in [1], and certain aspects of it for the general four-manifold $X$ have been worked out in [12] and also recently in [16], [17], [28].

\subsection{LOW-ENERGY THEORY}

The low-energy theory contains $r \mathcal{N}=2$ vector multiplets, which are defined up to $\Gamma$ - transformation, where $\Gamma$ is a subgroup of $S p_{2 r}(\mathbb{Z})$, e.g $\Gamma(2)$ or $\Gamma_{0}(4)$ for $r=1$. Let us denote the scalar components of the multiplet, which are monodromy invariant (up to a sign) at $u^{k}=\infty$ by $a^{i}$. Then the $S$-dual ones will be denoted as $a_{i, D}$. The low-energy effective couplings are denoted as $\tau_{i j}(a)=\left(\frac{4 \pi i}{g_{e f f}^{2}}+\frac{\theta_{e f f}}{2 \pi}\right)_{i j}=\frac{\partial a_{i, D}}{\partial a^{j}}=\tau_{i j, 1}+i \tau_{i j, 2} \equiv \Re \mathrm{e} \tau_{i j}+i \Im \mathrm{m} \tau_{i j}$

The $Q$ - transformations of the gauge fields must be consistent with the electric-magnetic duality. It is not clear a priori that such a $Q$-action exists, since the duality is a non-local operation on the fields, while the $Q$ is a local one. One may try to imitate the twisted version of the supersymmetric duality transformation presented in [1].

We take the approach where only holomorphuic representatives of the fields are used. It allows for a quick check of the modular invariance of the measure in a relatively simple setting. One has to work with (formal) contour integrals. Most of the constructions can be done working with the "holomorphic" fields $a, \psi, A$ only. The only trouble with such a prescription is the absence of Laplacians and non-definiteness of the topological terms like $F \wedge F$. The first problem is avoided in certain cases by working with cohomology (with harmonic forms) while the second may be treated via analytic continuation. In any case such an approach is useful in getting the right structures. Once it is done one may introduce the quartet of the fields $\bar{a}, \eta, \chi, H$ and justify the constructions by working with the standard positive-definite actions.

\footnotetext{
${ }^{1}$ They are honest solutions of instanton equations with abelian gauge group

${ }^{2}$ The contact term between the two-observables constructed out of quadratic casimirs and certain steps in extending the integrals of [16] to the case of $S U(N)$ gauge group are independently obtained in [28]
} 
Consider the short superfield:

$$
\mathcal{A}_{i, D}=a_{i, D}+\psi_{i, D}+F_{i, D}
$$

where $d F_{i, D}=0$. The operator $Q$ acts as follows: $Q a_{i, D}=0, Q \psi_{i, D}=d a_{i, D}$, $Q F_{D}=d \psi_{i, D}$. We impose (by hands) the condition that $F_{i, D}$ represents the integral cohomology class of the space-time manifold $\Sigma$. Thus, $\mathcal{A}_{i, D} \in$ $\Omega^{0}(\Sigma)_{B} \oplus \Omega^{1}(\Sigma)_{F} \oplus \Omega_{\mathbb{Z}}^{2}(\Sigma)_{B}$ Here $\Omega^{2}(\Sigma)_{\mathbb{Z}}$ is the space of closed two-forms with periods in $2 \pi i \mathbb{Z}$. The indices $B, F$ denote the bosonic and fermionic fields respectively. The superfield $\mathcal{A}_{D, i}$ obeys the condition $(Q-d) \mathcal{A}_{D, i}=0$. One may also fulfill the condition of $Q-d$-closedeness by introducing a complete set of $p$-forms which we call the long superfield: $\mathcal{A}^{i}=\sum_{p=0}^{4} \mathcal{A}^{i, p} \in$

TABLE 2. Long superfield

\begin{tabular}{llllll}
\hline Degree of a form & 0 & 1 & 2 & 3 & 4 \\
\hline Field $\mathcal{A}^{i}=$ & $a^{i}+$ & $\psi^{i}+$ & $F^{i}+$ & $\rho^{i}+$ & $D^{i}$ \\
\hline
\end{tabular}

$V=\oplus_{p=0}^{4} \Omega^{p}(X)$ and $Q$ acts as $Q \mathcal{A}^{i, p}=d \mathcal{A}^{i, p-1}$. Let $\mathcal{F}_{D}$ be a holomorphic function on $\mathbb{C}^{r}$. The "action"

$$
S=\int_{\Sigma} \mathcal{F}_{D}\left(\mathcal{A}_{D}\right)
$$

is clearly $Q$-invariant. The long superfield $\mathcal{A}$ allows reparameterizations:

$$
\mathcal{A}^{i} \mapsto \widetilde{\mathcal{A}^{i}}(\mathcal{A})
$$

induced by the holomorphic maps $a^{i} \mapsto \tilde{a}^{i}\left(a^{k}\right)$. Let $\mathcal{L} \subset \mathbb{C}^{2 r}$ be a $\Gamma$-invariant Lagrangian subvariety. Let $u^{k}, k=1, \ldots r$ be the generators of the ring $\mathcal{W}_{\mathcal{L}}$ of globally defined $\Gamma$-invariant holomorphic functions on $\mathcal{L}$. Extend them to the long superfields $\mathcal{U}^{k}, k=1, \ldots, r$. Define the measure

$$
[\mathcal{D U}]=\prod_{k=1}^{r} d u^{k} d \psi_{u}^{k} d F_{u}^{k} d \rho_{u}^{k} d D_{u}^{k}
$$

where $(Q-d)\left(u^{k}+\psi_{u}^{k}+F_{u}^{k}+\rho_{u}^{k}+D_{u}^{k}\right)=0$. The duality transformation proceeds as follows: introduce both $\mathcal{A}_{i, D}$ and $\mathcal{A}^{i}$ and consider the action $S^{\prime}=\int_{\Sigma} \mathcal{A}^{i} \mathcal{A}_{i, D}-\mathcal{F}\left(\mathcal{A}^{i}\right)$. Let us consider the following (formal) path integral:

$$
\int \mathcal{D} \mathcal{U}^{i} \mathcal{D} \mathcal{A}_{i, D} e^{-S}
$$


The measure $\mathcal{D} \mathcal{A}_{i, D}$ is defined canonically. The dependence of the measure on $\mathcal{A}^{i}$ on the choice of the measure $d u^{1} \wedge \ldots d u^{r}$ is completely parallel to anomaly in Type $\mathbf{B}$ sigma models in two dimensions [19],[11]. The integral over $\mathcal{A}_{i, D}$ (together with summation over the fluxes of $F_{i, D}$ ) forces $D^{i}, \rho^{i}$ to vanish, while $F^{i}$ becomes a curvature of a connection $A^{i}$. As a result one gets a measure

$$
\operatorname{Det}_{i j}\left(\frac{\partial u^{i}}{\partial a^{j}}\right)^{\frac{\chi}{2}} \prod_{k=1}^{r} d a^{k} d \psi^{k} d F^{k}
$$

On the other hand, performing the integral over $\mathcal{U}$ gives us: $a_{i, D}=\frac{\partial \mathcal{F}}{\partial a^{i}}$, $\psi^{i}=\left(\tau^{-1}\right)^{i j} \psi_{j, D}, F^{i}=\left(\tau^{-1}\right)^{i j}\left(F_{j, D}-\frac{1}{2}\left(\tau^{-1}\right)^{l m}\left(\tau^{-1}\right)^{k p}\left(\partial_{l k j}^{3} \mathcal{F}\right) \psi_{m, D} \psi_{p, D}\right)$ with $\tau_{i j}=\frac{\partial^{2} \mathcal{F}}{\partial a^{i} \partial a^{j}}$. The determinants in this case are slightly involved:

$$
\begin{gathered}
\operatorname{Det}_{i j}\left(\frac{\partial u^{i}}{\partial a^{j}}\right)^{\frac{\chi}{2}}(\operatorname{Det} \tau)^{-\operatorname{dim} \Omega^{0}+\operatorname{dim} \Omega^{1}-\frac{1}{2} \operatorname{dim} \Omega^{2}}= \\
(\operatorname{Det} \tau)^{-\frac{\chi}{2}} \operatorname{Det}_{i j}\left(\frac{\partial u^{i}}{\partial a^{j}}\right)^{\frac{\chi}{2}}= \\
\operatorname{Det}_{i j}\left(\frac{\partial u^{i}}{\partial a_{D, j}}\right)^{\frac{\chi}{2}}
\end{gathered}
$$

Were there no factor $\operatorname{Det}_{i j}\left(\frac{\partial u^{i}}{\partial a^{j}}\right)^{\frac{\chi}{2}}$ the duality transformation would be anomalous. This anomaly was already observed in [12] (for $r=1$ ). The "action" $S^{\prime}$ evaluates to (13) with the substitution of $\mathcal{F}$ by $\mathcal{F}_{D}$, which is the Legendre transform of $\mathcal{F}$.

As it has been explained in [12] the low-energy effective action contains the terms which account for the coupling to the background gravitational field:

$$
e^{S_{\text {grav }}} \sim e^{b(u) \chi+c(u) \sigma}
$$

Here $b(u), c(u)$ are the gravitational renormalization coefficients computed for the low-energy $S U(2)$ theory in [12]. We already know that

$$
e^{2 b}=\operatorname{Det} \frac{\partial u^{k}}{\partial a^{l}}
$$

It remains to compute $e^{c}$. Imagine that the manifold $X$ is replaced by a blowup $\widetilde{X}$ at the point $P$. Geometrically it means that we glue a copy of $\overline{\mathbb{P}}^{2}$ to $X$. The Euler characteristics and the signature of the manifold $\widetilde{X}$ are $\chi+1$ and $\sigma-1$ respectively since $b_{2}^{-}$is increased by one in the process $\left(\overline{\mathbb{P}}^{2}\right.$ contains a non-contractible two-sphere with self-intersection -1 ). Consider an integral of the the divergence of the ghost number current over the glued $\overline{\mathbb{P}}^{2}$ in the instanton sector with the total instanton number $k$. The instanton 
charge splits as a sum $k=k_{1}+k_{2}$ where $k_{1}$ is what is left on $X-P$, and $k_{2}$ is what has gone to $\overline{\mathbb{P}}^{2}$. The divergence picks up an anomaly:

$$
\int_{\overline{\mathbb{P}}^{2}} d J=2 \beta_{1} k_{2}
$$

where $\beta_{1}$ is the perturbative beta-function. In the case of pure gauge theory it is equal to $-2 h^{\vee}, h^{\vee}$ being the dual Coxeter number. There also could be a gravitational contribution to (19) but it vanishes because $b_{2}^{+}\left(\overline{\mathbb{P}}^{2}\right)=0$. Now, using factorization we may replace the glued $\overline{\mathbb{P}}^{2}$ by a local operator $\mathcal{B}\left(u^{k}\right)$ which must produce the same anomaly as in (19). If the theory is asymptotically free, then the right hand side of (19) is negative for $k_{2}>$ 0 and therefore the operator $\mathcal{B}=1$ and only $k_{2}=0$ contributes. The conclusion is that the blowup does not change the measure. On the other hand the gravitational renormalization together with extra piece of Maxwell partition function combine to

$$
e^{b-c} \Theta(\tau)=1
$$

where $\Theta(\tau)$ is a certain theta-constant which is discussed below. Hence:

$$
e^{S_{\text {grav }}} \sim \operatorname{Det}_{i j}\left(\frac{\partial u^{i}}{\partial a^{j}}\right)^{\frac{\chi+\sigma}{2}} \Theta(\tau)^{\sigma}
$$

It remains to notice that

$$
\operatorname{Det}_{i j}\left(\frac{\partial u^{i}}{\partial a^{j}}\right)^{4} \Theta^{8}=\Delta\left(u_{i}\right)
$$

is the modular invariant function on the moduli space of vacua (the discriminant) and rewrite (21) as

$$
e^{S_{\text {grav }}} \sim \operatorname{Det}_{i j}\left(\frac{\partial u^{i}}{\partial a^{j}}\right)^{\frac{\chi}{2}} \Delta^{\frac{\sigma}{8}}
$$

There is another gravitational correction to the effective action, described in [12], namely, if the manifold $X$ is not spin, then there is a term $e^{\frac{1}{2}\left(w_{2}(X), F\right)}$ in the effective measure. We can get rid of it in the course of the study of $S U(2)$ theory by the shift $\tau \rightarrow \tau+1$ thanks to $\mathrm{Wu}$ formula. The sign generalizes to

$$
e^{\left(\langle F, \rho\rangle, w_{2}(X)\right)}
$$

in the case of general simply-laced group. Suppose we are to integrate the massive $W$-bosons out. Let $a \in \underline{\mathbf{t}}$ be the scalar in the Cartan part of the 
vector multiplet. The one-loop determinants on bosons and fermions cancel on non-zero modes leaving purely holomorphic contribution:

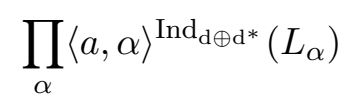

where $\alpha$ runs over the set of roots, $L_{\alpha}$ is the line bundle corresponding to the root $\alpha, \operatorname{Ind}_{\mathrm{d} \oplus \mathrm{d}^{*}}\left(\mathrm{~L}_{\alpha}\right)$ is the index of the operator $d \oplus d^{*}$ coupled to the line bundle $L_{\alpha}$ (the $W_{\alpha}$-boson multiplet). The index formula gives $\operatorname{Ind}_{d \oplus d^{*}}\left(L_{\alpha}\right)=\frac{1}{2}\left(\int_{X} c_{1}\left(L_{\alpha}\right)^{2}+c_{1}(X) c_{1}\left(L_{\alpha}\right)\right)+\frac{\chi+\sigma}{4}$. We see that the terms $c_{1}\left(L_{\alpha}\right)^{2}$ collect into the perturbative beta-function, the term proportional to $\frac{\chi+\sigma}{4}$ yields the asymptotics of $(22)$ in the $\Lambda \rightarrow 0$ limit and the odd part $c_{1}(\Sigma) c_{1}\left(L_{\alpha}\right)$ survives in the form $\prod_{\alpha>0}(-1)^{c_{1}(X) c_{1}\left(L_{\alpha}\right)} \equiv e^{\left(\langle F, \rho\rangle, w_{2}(X)\right)}$ since $c_{1}(X) \equiv w_{2}(X) \bmod 2$. We denoted by $c_{1}(X)$ the first Chern class of the canonical bundle of the almost complex structure which exists on any four-manifold (cf. [12]).

\subsection{SPECIFIC COMPUTATIONS OF THE CONTACT TERMS}

\subsubsection{Two-observables}

Suppose we are interested in computing $\left\langle\exp \int_{C_{a}} \mathcal{O}_{\mathcal{P}_{a}}^{(2)}\right\rangle_{X}$, where $C_{a}$ are twocycles on a four-manifold $X$ which may intersect. If we go ahead and write down the effective low-energy measure we immediately face the problem of modular anomaly. Indeed, the low-energy measure contains a theta-function $\Theta_{H_{2}(X ; \mathbb{Z})}(\tau)^{3}$ associated to an intersection form of $X$. It comes from the partition function of the Maxwell theory. In the presence of the two-observables this theta-function has an argument: $\Theta(\vec{z}, \tau), z_{i}=C_{a}^{\vee} \frac{\partial \mathcal{P}_{a}}{\partial a^{i}}$ and its modular transformation produces a factor

$$
\exp \sum_{a, b} \#\left(C_{a} \cap C_{b}\right)\left(\tau^{-1}\right)^{i j} \frac{\partial \mathcal{P}_{a}}{\partial a^{i}} \frac{\partial \mathcal{P}_{b}}{\partial a^{j}}
$$

This factor must be cancelled by an anomaly of an additional interaction which is developed due to the presence of intersecting densities, i.e. due to contact terms.

In order to derive this interaction we again use the trick with blow up of a manifold $X$ this time at the intersection point $P$. The homology lattice $H_{*}(\widetilde{X})$ of $\widetilde{X}$ is that of $X$ plus a factor of $\mathbb{Z}$. The intersection form is simply

$$
(,)_{\tilde{X}}=(,)_{X} \oplus(-1)
$$

\footnotetext{
${ }^{3}$ in the harmonic approach it becomes Siegel theta-function associated to a lattice of signature $\left(b_{2}^{+}, b_{2}^{-}\right)$
} 
as the exceptional divisor $e$ (the two-sphere inside $\overline{\mathbb{P}}^{2}$ ) has self-intersection -1 . Under the isomorphism $H_{*}(\tilde{X})=H_{*}(X) \oplus \mathbb{Z}$ the inverse images of the cycles in $X$ belong to the component $H_{*}(X)$ of $H_{*}(\widetilde{X})$. We shall denote them by the same letters as the cycles in $X$. To derive the contact term we compare the correlation functions $\left\langle\int_{C_{1}} \mathcal{O}_{\mathcal{P}_{1}}^{(2)} \int_{C_{2}} \mathcal{O}_{\mathcal{P}_{2}}^{(2)} \ldots\right\rangle_{X}$ and $\left\langle\int_{\tilde{C}_{1}} \mathcal{O}_{\mathcal{P}_{1}}^{(2)} \int_{\tilde{C}_{2}} \mathcal{O}_{\mathcal{P}_{2}}^{(2)} \ldots\right\rangle_{\widetilde{X}}$ where the cycles $\tilde{C}_{1}, \tilde{C}_{2} \in H_{*}(\widetilde{X})$ do not intersect each other in the vicinity of $P$ and are given by the formulae:

$$
\tilde{C}_{k}=C_{k}-e, \quad \# \tilde{C}_{1} \cap \tilde{C}_{2}=\# C_{1} \cap C_{2}-1
$$

Consider the same ghost number current integral as in (19). Due to the presence of two 2-observables $\int_{e} \mathcal{O}_{\mathcal{P}_{1,2}}^{(2)}$ the anomaly changes to:

$$
\int_{\overline{\mathbb{P}}^{2}} d J=2 \beta_{1} k_{2}+U\left(\mathcal{P}_{1}\right)+U\left(\mathcal{P}_{2}\right)-4
$$

where $U(\ldots)$ denotes the ghost charge which equals twice the degree of $\ldots$ for homogeneous $\mathcal{P}_{1,2}$. For simply-laced group and for $k_{2}>0$ the right hand side of $(27)$ does not exceed $2(2 h-2)-2\left(2 h-T_{m}\right)=2 T_{m}-4$, where $T_{m}$ is the contribution of matter to the perturbative beta-function. Assuming that $T_{m}<2$ we see that again the operator $\mathcal{B}$ must be equal to one (see [21], [25] for mathematical proof of this result). The net effect of our manipulations is the replacement of the intersecting cycles on the manifold $X$ by the non-intersecting cycles on the manifold $\tilde{X}$. Physically the crucial fact is that under the blowup of a point $P$ a new two-cycle $e$ appears and it leads to the possibility for the gauge field to have a flux through it. In the low-energy effective theory the insertion of the new two-cycle must be reflected in the new factor in the Maxwell partition function, which is the sum over all line bundles on $\overline{\mathbb{P}}^{2}$ in the presence of two 2-observables $\int_{e} \mathcal{O}_{\mathcal{P}_{1,2}}^{(2)}$ This new factor is simply:

$$
\frac{\partial \mathcal{P}_{1}}{\partial a^{i}} \frac{\partial \mathcal{P}_{2}}{\partial a^{j}} \frac{\partial}{\partial \tau_{i j}} \log \Theta(\tau)
$$

where

$$
\Theta=\sum_{\lambda \in \Lambda} \exp (2 \pi i\langle\lambda, \tau \lambda\rangle+\pi i\langle\lambda, \rho\rangle)
$$

with $\Lambda$ being the set of weights and $\langle$,$\rangle the restriction of the Killing form$ on $\underline{\mathbf{t}}$ - the Cartan subalgebra of $\mathbf{g}$. The term $(-)^{\langle\lambda, \rho\rangle}$ is $(23)$ specified to $\overline{\mathbb{P}}^{2}$ case. The numerator $\partial_{\tau} \Theta$ comes from evaluating the sum over fluxes in the presence of two-observables while the denominator $\Theta^{-1}$ is the remnant of the gravitational renormalization factors $e^{b-c}$ from (20). Summarizing, we 
have shown that in the theories with $T_{m}<2$ the pair contact term is equal to (and more generally if $U\left(\mathcal{P}_{1}\right)+U\left(\mathcal{P}_{2}\right)-4+2 \beta_{1}<0$ ):

$$
\mathcal{C}\left(\mathcal{P}_{1}, \mathcal{P}_{2}\right)=\frac{\partial \mathcal{P}_{1}}{\partial a^{i}} \frac{\partial \mathcal{P}_{2}}{\partial a^{j}} \frac{\partial}{\partial \tau_{i j}} \log \Theta(\tau)
$$

\subsection{CONTACT TERM OF 0-OBSERVABLES AND 4-OBSERVABLES.}

Another way of getting the contact term $\mathcal{C}\left(\mathcal{P}_{1}, \mathcal{P}_{2}\right)$ is by considering 4 - and 0 -observables. Let us consider asymptotically free theory with massless matter. Let $\mathcal{P}_{1}=u_{1}$ be the generator whose fourth decsendant produces the instanton charge (quadratic casimir). The insertion of $e^{t \int_{X} u_{1}^{(4)}}$ is equivalent to multiplication of the correlator in the sector with instanton charge $k$ by $e^{2 \pi i t k}$. It is possible to show that this manipulation is in turn equivalent to rescaling of the fields $u_{k} \rightarrow e^{\frac{2 \pi i d_{k}}{\beta_{1}} t} u_{k}$ and $a^{i} \rightarrow e^{\frac{4 \pi i t}{\beta_{1}}} a^{i}$, where $d_{k}$ are the weights of the homogeneous generators $u_{k}$ of the ring of invariant polynomials. This idea yields the following formula [17]:

$$
\mathcal{C}\left(u_{1}, u_{k}\right)=\frac{4 \pi i}{\beta_{1}}\left(a^{i} \frac{\partial u_{k}}{\partial a^{i}}-d_{k} u_{k}\right)
$$

Comparing (31) and (30) in cases where it is possible we get an interesting identity, which singles out the family of SW curves and their generalizations [1], [2],[3]. For concreteness we consider $S U\left(N_{c}\right)$ theory with fundamental massless matter:

$$
\frac{\partial u_{1}}{\partial a^{i}} \frac{\partial u_{k}}{\partial a^{j}} \frac{\partial}{\partial \tau_{i j}} \log \Theta(\tau)=\frac{1}{2 N_{c}-N_{f}}\left(a^{i} \frac{\partial u_{k}}{\partial a^{i}}-(k+1) u_{k}\right)
$$

where $N_{f}$ is the number of matter hypermultiplets $a^{i}$ are the $A$-periods of the differential $x \frac{d z}{z}$ on the curve:

$$
z+\frac{x^{N_{f}}}{z}=x^{N_{c}}-\sum_{k=1}^{N_{c}-1} u_{k} x^{N_{c}-k-1}
$$

The formula (32) must be valid for $N_{f}<N_{c}$ as follows from the arguments under the formula (27) applied to the case $\mathcal{P}_{1}=u_{1}, \mathcal{P}_{2}=u_{k}$ as in this case $U\left(\mathcal{P}_{1}\right)+U\left(\mathcal{P}_{2}\right)-4=2 k+2 \leq 2 N_{c}$ while $2 \beta_{1} k_{2} \leq 2\left(N_{f}-2 N_{c}\right)<-2 N_{c}$.

The formula (32) follows from the modular properties and the asymptotics at $u \rightarrow \infty$ : both left and right hand sides of (32) vanish in the limit $u \rightarrow \infty$. The modular properties of the left hand side follow easily from 
the theta constant behavior while the right hand side enjoys the following property:

$$
\begin{gathered}
\left.\frac{2 \pi i}{2 N_{c}-N_{f}}\left(a^{i} \frac{\partial u_{k}}{\partial a^{i}}-(k+1) u_{k}\right)\right|_{D}-\frac{2 \pi i}{2 N_{c}-N_{f}}\left(a^{i} \frac{\partial u_{k}}{\partial a^{i}}-(k+1) u_{k}\right)= \\
=\frac{2 \pi i}{2 N_{c}-N_{f}}\left(a_{D, j}-a^{i} \tau_{i j}\right) \frac{\partial u_{k}}{\partial a^{i}}\left(\tau^{-1}\right)^{i j}= \\
=\frac{1}{2} \frac{\partial u_{k}}{\partial a^{i}} \frac{\partial u_{1}}{\partial a^{j}}\left(\tau^{-1}\right)^{i j}
\end{gathered}
$$

thanks to the formulae of [18].

It seems possible to promote the formulae for the contact terms (30) and (31) to the evolution equations a la Whitham hierarchy (cf. [8], [9]). Details will be shown in a separate publication [4].

\section{Remarks on ADHM integration}

Consider the moduli space $\mathcal{M}_{k, N}$ of charge $k U(N)$ instantons on $\mathbb{R}^{4}$. By ADHM construction it is the hyperkahler quotient of linear space of dimension $4 k^{2}+4 k N$ by the action of $U(k)$. That is, it is the quotient of the space of solutions of certain equations $\vec{\mu}=0$ by $U(k)$. This representation allows to get the expressions for the integrals of cohomology classes over $\mathcal{M}_{k, N}$ in terms of the contour integrals over the complexified Lie algebra of $U(k)$. The integrals are localized according to the standard equivariant techniques [10] to the fixed points of $U(k)$ action which are nothing but the point-like instantons! The space of those is non-compact - the instanton can run away to infinity. In order to cure this problem let us take into account the natural actions of the Lorentz group $S O(4)$ and gauge group $U(N)$. Suppose we compute the integrals over $\mathcal{M}_{k, N}$ of the $S O(4) \times U(N)$-equivariant cohomology classes $\omega_{1}(\vec{\epsilon} ; \vec{a}), \ldots, \omega_{p}(\vec{\epsilon} ; \vec{a})$. Here $\vec{\epsilon}=\left(\begin{array}{c}\epsilon_{1} \\ \epsilon_{2}\end{array}\right)$ is the generator of the Cartan subalgebra of $S O(4)$ and $\vec{a}=\left(a_{1}, \ldots, a_{N}\right)$ is the generator of $U(N)$ Cartan subalgebra. $\vec{\epsilon}$ generates rotations in two orthogonal planes in $\mathbb{R}^{4}$. The fixed points of such rotation are formed by the instantons which sit on top of each other in the center of rotation. Now the integral can be evaluated (cf. [29]). The classes $\omega_{l}$ are in one-to-one correspondence with the invariant polynomials $P_{l}$ on the Lie algebra of $U(k)$, i.e. symmetric functions in $k$ variables. We claim that

$$
\begin{gathered}
Z(\vec{\epsilon} ; \vec{a}):=\int_{\mathcal{M}_{k, N}} \prod_{l} \omega_{l}(\vec{\epsilon} ; \vec{a})= \\
\frac{\left(\epsilon_{1}+\epsilon_{2}\right)^{k}}{\epsilon_{1}^{k} \epsilon_{2}^{k}} \oint \bigwedge_{i=1}^{k} \frac{d z_{i}}{\prod_{\lambda}\left(z_{i}+a_{\lambda}\right)\left(z_{i}+a_{\lambda}+\epsilon_{1}+\epsilon_{2}\right)} \quad \prod_{i \neq j} \frac{z_{i j}\left(z_{i j}+\epsilon_{1}+\epsilon_{2}\right)}{\left(z_{i j}+\epsilon_{1}\right)\left(z_{i j}+\epsilon_{2}\right)}
\end{gathered}
$$


where $z_{i j}=z_{i}-z_{j}$.

The formula (33) is the only sensible calculation one may perform using ADHM data. To compare it with the calculations involving SW low-energy effective action one needs to develop a formalism which takes into account the isometries of the space-time manifold. It is equivalent to working with $Q+\epsilon^{\mu} G_{\mu}$ - cohomology instead of $Q$-cohomology (cf. [6], [20]). This theory is currently under study [4].

\section{Acknowledgements}

We thank G. Moore for many discussions on Donaldson theory and related matters over the years. S.Sh. is grateful to E. Witten for important discussion reviving the interest in the subject. We are grateful to A. Gerasimov, A.Gorsky, A. Lawrence, A. Marshakov, A. Morozov, A. Mironov, N. Seiberg and C. Vafa for useful discussions.

The research of A. L. is supported partially by DOE under grant DEFG02-92ER40704, by PYI grant PHY-9058501 and RFFI under grant 9601-01101. The research of N. N. is supported by Harvard Society of Fellows, partially by NSF under grant PHY-92-18167, and partially by RFFI grant 96-02-18046. The research of S.Sh. was supported by DOE grant DE-FG0292ER40704, by NSF CAREER award, by OJI award from DOE and by A.P. Sloan Foundation. A. L. and N. N. are also supported by grant 96-15-96455 for scientific schools.

\section{References}

1. N. Seiberg, E. Witten, "Electric-Magnetic Duality, Monopole Condensation, And Confinement in $N=2$ Supersymmetric Yang-Mills Theory" Nucl. Phys. B426 (1994) 19-52 (and erratum - ibid. B430 (1994) 485-486 )

"Monopoles, Duality and Chiral Symmetry Breaking in $\mathrm{N}=2$ Supersymmetric QCD", hep-th/9408099, Nucl. Phys. B431 (1994) 484-550.

2. P.C. Argyres, A.E. Farragi, "The Vacuum Structure and Spectrum of $N=2$ Supersymmetric $S U(n)$ Gauge Theory", hep-th/9411057

A. Klemm, W. Lerche, S. Theisen and S. Yankielowicz, "Simple Singularities and $\mathcal{N}=2$ Supersymmetric Yang-Mills Theory", hep-th/9411048

3. P.C. Argyres, M.R. Plesser and N. Seiberg, "The Moduli Space of Vacua of $N=2$ SUSY QCD and Duality in $N=1$ SUSY QCD", hep-th/9603042

4. A. Losev, N. Nekrasov, S. Shatashvili, in progress

5. N. Dorey, V. Khoze, M. Mattis, "Supersymmetry and Multi-Instanton Measure", hep-th/9708036

6. A. Losev, Theor.Math.Phys. 95 (1993) 595

7. K. Saito, Publ. RIMS, Kyoto Univ. 19 (1983) 1231

B. Blok, A. Varchenko, Int.J.Mod.Phys.A7 (1992) 1467-1490

R. Dijkgraaf, talk on RIMS conference "Infinite dimensional analysis", July 1993

A. Losev, in "Integrable models and Strings ", 172, Proceedings of 1993 Helsinki Conference, Springer-Verlag, 1995

A. Losev, I. Polyubin, Int.J.Mod.Phys. A10 (1995) 4161 
8. A. Gorsky, I. Krichever, A. Marshakov, A. Morozov, A. Mironov, hepth/9505035, Phys.Lett.B355: 466-474, 1995

9. I. Krichever, in "30 years of the Landau Institute", 477 , I.M. Khalatnikov, V.P. Mineev, V.P. (eds.)

10. M. Atiyah, R. Bott, Topology 23 (1984) 1-28

11. E. Witten, "Introduction to Cohomological Field Theories", Int. J. Mod. Phys. A6 (1991) 2775.

12. E. Witten, Selecta Mathematica 1 (1995) 383

13. E. Witten, "Topological Quantum Field Theory", Commun. Math. Phys. 117 (1988) 353.

14. E. Witten, "Monopoles and Four-Manifolds", hep-th/9411102

15. E. Witten, "On Quantum gauge theories in two dimensions," Commun. Math. Phys. 141 (1991) 153

"Two dimensional gauge theories revisited", J. Geom. Phys. 9 (1992) 303-368

16. G. Moore, E. Witten, "Integration over $u$-plane in Donaldson theory", hepth/9709193

17. A. Losev, N. Nekrasov, S. Shatashvili, "Issues in topological gauge theory", hepth/9711108, YCTP-P22/97, HUTP-97/A064, ITEP-TH-49/97

18. M. Matone, "Instantons and recursion relations in $\mathcal{N}=2$ susy gauge theory", hepth/9506102, Phys. Lett. B357 (1995) 342

19. S. Cecotti, C. Vafa, "Topological Anti-Topological Fusion", Nucl. Phys. B367 (1991) 359

20. A. Givental, alg-geom/9603021, alg-geom/9701016

21. R. Friedman, J. Morgan, "Smooth Four-Manifolds and Complex Surfaces", Ergebnisse der. Math. und Grenz. 3, Springer-Verlag, New York, 1994

22. L. Göttsche, alg-geom/9506018

23. L. Göttsche, D. Zagier, alg-geom/9612020

24. R. Borcherds, alg-geom/9609022

25. R. Fintushel and R.J. Stern, Annals of Math. 143(1996) 529

26. A. Gerasimov, hepth $/ 9305090$

27. M. Blau, G. Thompson, Nucl. Phys. B408 (1993) 345-390

28. M.Mariño, G. Moore, "Integrating over the Coulomb branch in $\mathcal{N}=2$ gauge theory", hep-th/9712062

29. G. Moore, N. Nekrasov, S. Shatashvili, "Integrating over Higgs branches", hepth/9712241, HUTP-97/A089, YCTP-P23/97, ITEP-TH-62/97 